\section{A Classificação Internacional de Funcionalidade, Incapacidade e Saúde da Organização Mundial da Saúde: Conceitos, Usos e Perspectivas}

\section{The International Classification of Functioning, Disability and Health: Concepts, Uses and Perspectives}

\section{Norma Farias ${ }^{1 *}$}

\section{Cassia Maria Buchalla²}

${ }^{1}$ Instituto de Saúde - Secretaria de Estado da Saúde de São Paulo. São Paulo, SP, Brasil.

${ }^{2}$ Departamento de Epidemiologia- Faculdade de Saúde Pública da Universidade de São Paulo. São Paulo, SP, Brasil

*Correspondência: Norma Farias. Instituto de Saúde, Rua Santo Antônio, 590, 01314-000 São Paulo, SP, Brasil. E-mail: norma@isaude.sp.gov.br

\section{Resumo}

O objetivo do presente artigo é apresentar a Classificação Internacional de Funcionalidade, Incapacidade e Saúde (CIF), que faz parte da "família" de classificações desenvolvida pela Organização Mundial da Saúde (OMS). São apresentados o histórico e o processo de revisão da classificação anterior Classificação Internacional de Deficiências, Incapacidades e Desvantagens (CIDID)- que deram origem à atual classificação - CIF. O modelo da CIF substitui o enfoque negativo da deficiência e da incapacidade por uma perspectiva positiva, considerando as atividades que um indivíduo que apresenta alterações de função e/ou da estrutura do corpo pode desempenhar, assim como sua participação social. A funcionalidade e a incapacidade dos indivíduos são determinadas pelo contexto ambiental onde as pessoas vivem. A CIF representa uma mudança de paradigma para se pensar e trabalhar a deficiência e a incapacidade, constituindo um instrumento importante para avaliação das condições de vida e para a promoção de políticas de inclusão social. A classificação vem sendo incorporada e utilizada em diversos setores da saúde e equipes multidisciplinares. No entanto, será mais adequada à medida que for utilizada por um número maior de profissionais, em locais diversos e a partir de pessoas e realidades diferentes.

Palavras-chave: Classificação Internacional de Funcionalidade, Incapacidade e Saúde. Organização Mundial da Saúde. Pessoas com deficiência. 
Abstract

The aim of this article is to present the International Classification of Functioning, Disability and Health (ICF), which comprises the "family" of classifications of the World Health Organization (WHO). This study presents the background and the process of reviewing the previous classification, the International Classification of Impairments, Disabilities, and Handicaps (ICIDH), on which the current classification was based. The framework of ICF replaces the negative perspective of impairment and disability with a positive standpoint, considering the activities that a person with alterations in body functions and/or structures can perform, and also their social participation. The functioning and impairment are determined by the environmental context where people live. The ICF represents a change on the paradigm for thinking and working impairment and disability, establishing an important instrument to evaluate the living conditions and to improve social inclusion policies. The classification has been incorporated and is being used by different professionals in several healthcare sectors. Nevertheless, it will become more adequate as it is used by a greater number of professionals working in different contexts and with a wider range of patients.

Key Words: International Classification of Functioning. Disability and Health. World Health Organization. Persons with impairment.
Uma das missões da Organização Mundial da Saúde - OMS consiste na produção de Classificações Internacionais de Saúde que representam modelos consensuais a serem incorporados pelos Sistemas de Saúde, gestores e usuários, visando a utilização de uma linguagem comum para a descrição de problemas ou intervenções em saúde ${ }^{1}$.

O propósito de "A Família de Classificações Internacionais” da OMS ( WHO Family of International Classifications - WHO-FIC) consiste em promover a seleção apropriada de classificações em vários campos da saúde em todo o mundo. Estas facilitam o levantamento, consolidação, análise e interpretação de dados; a formação de bases de dados nacionais consistentes, e permitem a comparação de informações sobre populações ao longo do tempo entre regiões e países ${ }^{1}$.

As condições de saúde relacionadas às doenças, transtornos ou lesões são classificadas na CID-10 (Classificação Estatística Internacional de Doenças e Problemas Relacionados à Saúde, $10^{a}$ Revisão) que fornece um modelo baseado na etiologia, anatomia e causas externas das lesões ${ }^{2}$. Dessa forma, a CID-10 constitui um instrumento útil para as estatísticas de saúde, tornando possível monitorar as diferentes causas de morbidade e de mortalidade em indivíduos e populações.

A necessidade de se conhecer o que acontece com os pacientes após o diagnóstico, com o decorrer do tempo, principalmente em relação às doenças crônicas e aos acidentes, torna-se cada vez mais importante para a área da saúde. Conhecer as causas de morte e as doenças mais freqüentes, em época que a expectativa de vida aumenta e a tecnologia ajuda a medicina a prolongar a vida humana, pode não ser suficiente para o planejamento de ações de saúde.

Esta comunicação tem como objetivo apresentar os pontos fundamentais desse novo modelo da Organização Mundial da Saúde, a Classificação Internacional de Funcionalidade, Incapacidade e Saúde, CIF. 


\section{Histórico da CIF}

Visando responder às necessidades de se conhecer mais sobre as conseqüências das doenças, em 1976 a OMS publicou a International Classification of Impairment, Disabilities and Handicaps (ICIDH), em caráter experimental. Esta foi traduzida para o Português como Classificação Internacional das Deficiências, Incapacidades e Desvantagens (handicaps), a CIDID ${ }^{3}$.

De acordo com esse marco conceitual, impairment (deficiência) é descrita como as anormalidades nos órgãos e sistemas e nas estruturas do corpo; disability (incapacidade) é caracterizada como as conseqüências da deficiência do ponto de vista do rendimento funcional, ou seja, no desempenho das atividades; handicap (desvantagem) reflete a adaptação do indivíduo ao meio ambiente resultante da deficiência e incapacidade ${ }^{4}$.

O modelo da CIDID descreve, como uma seqüência linear, as condições decorrentes da doença:

\section{Doença $\Rightarrow$ Deficiência $\Rightarrow$ Incapacidade $\Rightarrow$ Desvantagem}

O processo de revisão da ICIDH apontou suas principais fragilidades, como a falta de relação entre as dimensões que a compõe, a não abordagem de aspectos sociais e ambientais, entre outras. Após várias versões e numerosos testes, em maio de 2001 a Assembléia Mundial da Saúde aprovou a International Classification of Functioning, Disability and Health (ICF) $)^{5}$.

A versão em língua portuguesa foi traduzida pelo Centro Colaborador da Organização Mundial da Saúde para a Família de Classificações Internacionais em Língua Portuguesa com o título de Classificação Internacional de Funcionalidade, Incapacidade e Saúde, CIF .

\section{Conceituações, objetivos e componentes da CIF}

A CIF descreve a funcionalidade e a in- capacidade relacionadas às condições de saúde, identificando o que uma pessoa "pode ou não pode fazer na sua vida diária", tendo em vista as funções dos órgãos ou sistemas e estruturas do corpo, assim como as limitações de atividades e da participação social no meio ambiente onde a pessoa vive ${ }^{7,8}$.

Segundo a OMS, a CID-10 e a CIF são complementares: a informação sobre o diagnóstico acrescido da funcionalidade fornece um quadro mais amplo sobre a saúde do indivíduo ou populações. Por exemplo, duas pessoas com a mesma doença podem ter diferentes níveis de funcionalidade, e duas pessoas com o mesmo nível de funcionalidade não têm necessariamente a mesma condição de saúde ${ }^{9}$.

O termo do modelo da CIF é a funcionalidade, que cobre os componentes de funções e estruturas do corpo, atividade e participação social. A funcionalidade é usada no aspecto positivo e o aspecto negativo corresponde à incapacidade. Segundo esse modelo, a incapacidade é resultante da interação entre a disfunção apresentada pelo indivíduo (seja orgânica e/ou da estrutura do corpo), a limitação de suas atividades e a restrição na participação social, e dos fatores ambientais que podem atuar como facilitadores ou barreiras para o desempenho dessas atividades e da participação ${ }^{6,9}$.

A CIF é baseada, portanto, numa abordagem biopsicossocial que incorpora os componentes de saúde nos níveis corporais e sociais ${ }^{6,9}$. Assim, na avaliação de uma pessoa com deficiência, esse modelo destacase do biomédico, baseado no diagnóstico etiológico da disfunção, evoluindo para um modelo que incorpora as três dimensões: a biomédica, a psicológica (dimensão individual) e a social. Nesse modelo cada nível age sobre e sofre a ação dos demais, sendo todos influenciados pelos fatores ambientais (Figura 1). A OMS pretende incorporar também, no futuro, os fatores pessoais, importantes na forma de lidar com as condições limitantes.

O objetivo pragmático da CIF é fornecer uma linguagem padronizada e um modelo para a descrição da saúde e dos estados rela- 


\section{Condição de saúde \\ (transtorno ou doença)}

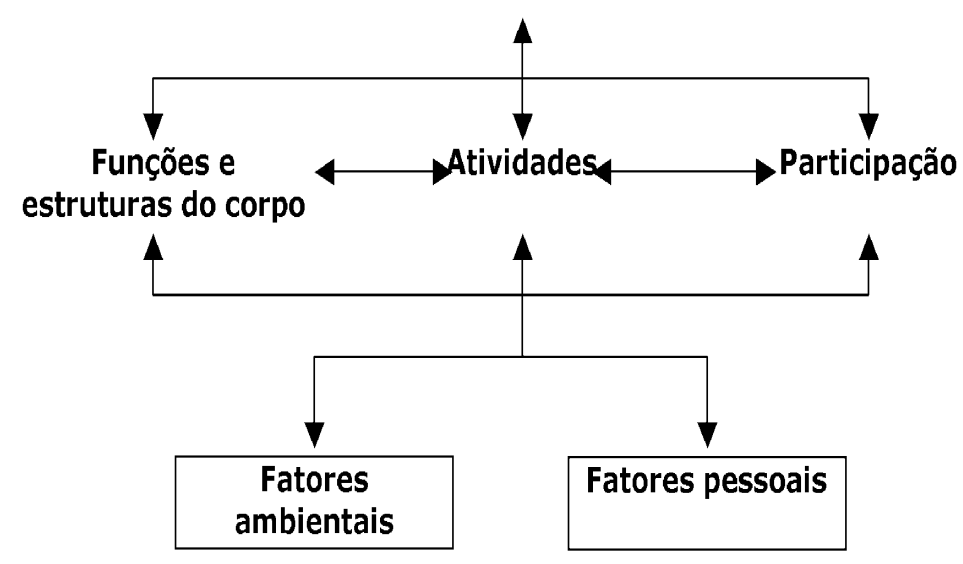

Figura 1 - Interação entre os componentes da CIF. Adaptação: OMS (2003) .

Figure 1 - Interaction between the components of ICF. Adapted from WHO (2003).

cionados à saúde, permitindo a comparação de dados referentes a essas condições entre países, serviços, setores de atenção à saúde, bem como o acompanhamento da sua evolução no tempo ${ }^{6}$.

No entanto, os conceitos apresentados na classificação introduzem um novo paradigma para pensar e trabalhar a deficiência e a incapacidade: elas não são apenas uma conseqüência das condições de saúde/doença, mas são determinadas também pelo contexto do meio ambiente físico e social, pelas diferentes percepções culturais e atitudes em relação à deficiência, pela disponibilidade de serviços e de legislação. Dessa forma, a classificação não constitui apenas um instrumento para medir o estado funcional dos indivíduos. Além disso, ela permite avaliar as condições de vida e fornecer subsídios para políticas de inclusão social.

\section{Terminologias utilizadas na CIF}

Os conceitos e terminologias utilizados na CIF são apresentados no Quadro 1.

As funções do corpo são definidas como as funções fisiológicas e psicológicas dos sistemas do corpo. As estruturas são definidas como as partes anatômicas do corpo, como os órgãos e seus componentes ${ }^{6,9}$.

As atividades e participação (A \& P) des- crevem como o indivíduo exerce suas atividades diárias e se engaja na vida social, considerando as funções e estruturas do seu corpo. O conteúdo desses componentes (A \& P) é organizado desde simples tarefas e ações até áreas mais complexas da vida, sendo incluídos itens referentes à aprendizagem e aplicação do conhecimento; tarefas e demandas gerais; comunicação, mobilidade, cuidados pessoais, atividades e situações da vida doméstica; relações e interações interpessoais; educação e trabalho; auto-suficiência econômica; vida comunitária ${ }^{6.9}$.

As limitações de atividade são as dificuldades que o indivíduo pode ter para executar uma determinada atividade. As restrições à participação social são os problemas que um indivíduo pode enfrentar ao se envolver em situações de vida ${ }^{6,9}$.

Os fatores ambientais constituem o "ambiente físico, social e de atitudes" em que as pessoas vivem e conduzem suas vidas. Esse componente inclui itens referentes a produtos e tecnologia; ambiente natural como clima, luz, som; apoios e relacionamentos como a família imediata, "cuidadores" e assistentes sociais; atitudes individuais e sociais; normas e ideologias; serviços, sistemas e políticas de previdência social, saúde, educação, trabalho, emprego, transportes, dentre outros ${ }^{6,9}$. 
Quadro 1 - Conceituações e terminologias dos componentes relatados na CIF.

Picture 1 - Concepts and terminology of the components listed in the ICF.

\begin{tabular}{|c|c|c|c|c|}
\hline Componente & $\begin{array}{l}\text { Funções do Corpo } \\
\text { Estruturas do Corpo }\end{array}$ & Atividade & Participação & Fatores Ambientais \\
\hline Definição & $\begin{array}{l}\text { Funções do corpo são } \\
\text { as funções fisiológicas } \\
\text { dos sistemas do corpo } \\
\text { ( incluindo as funções } \\
\text { mentais) . } \\
\text { Estruturas do corpo são } \\
\text { as partes anatômicas } \\
\text { do corpo. }\end{array}$ & $\begin{array}{l}\text { Atividade é a execução } \\
\text { de tarefas realizadas no } \\
\text { dia a dia de um } \\
\text { indivíduo. }\end{array}$ & $\begin{array}{l}\text { Participação é } \\
\text { o envolvimento } \\
\text { numa situação } \\
\text { da vida social. }\end{array}$ & $\begin{array}{l}\text { Compreende os } \\
\text { fatores externos } \\
\text { do meio ambiente } \\
\text { onde a pessoa } \\
\quad \text { vive. }\end{array}$ \\
\hline $\begin{array}{l}\text { Aspecto } \\
\text { Positivo }\end{array}$ & $\begin{array}{l}\text { Integridade } \\
\text { Funcional e } \\
\text { Estrutural }\end{array}$ & Atividade & Participação & Facilitadores \\
\hline
\end{tabular}

\begin{tabular}{|c|c|c|c|c|}
\hline \multicolumn{5}{|c|}{ FUNCIONALIDADE } \\
\hline $\begin{array}{l}\text { Aspecto } \\
\text { Negativo }\end{array}$ & Deficiência & $\begin{array}{l}\text { Limitação da } \\
\text { Atividade }\end{array}$ & $\begin{array}{l}\text { Restrição da } \\
\text { Participação }\end{array}$ & $\begin{array}{l}\text { Barreiras/ } \\
\text { Obstáculos }\end{array}$ \\
\hline
\end{tabular}

INCAPACIDADE

Fonte: adaptada de: (1) WHO.Towards a Common Language for Functioning, Disability and Health- ICF. Geneva, 2002 e (2) OMS: Classificação Internacional de Funcionalidade, Incapacidade e Saúde; coordenação da tradução: Cassia Maria Buchalla. São Paulo: EDUSP; 2003.

\section{Os Usos da CIF}

ACIF tem sido apontada como uma espécie de "canivete suíço": contém uma série de ferramentas e permite várias abordagens. Ela pode ser usada em muitos setores que incluem a saúde, educação, previdência social, medicina do trabalho, estatísticas, políticas públicas. Sua importância pode ser colocada para as práticas clínicas, ensino e pesquisa ${ }^{6,10}$.

$\mathrm{Na}$ área clínica, ela se propõe a servir de modelo de atendimento multidisciplinar, devendo servir para as várias equipes e os vários recursos de que dispõem os serviços, tais como médico, psicólogo, terapeuta, assistente social etc.

Uma das vantagens apontadas para a adoção do modelo é a possibilidade de uniformização de conceitos e, portanto, da utilização de uma linguagem padrão que permita a comunicação entre pesquisadores, gestores, profissionais de saúde, organizações da sociedade civil e usuários em geral ${ }^{6,9}$.

A diversidade de recursos se traduz na dificuldade de uso completo da mesma. Assim, como proposta de solução para facili- tar sua aplicação, têm sido criados instrumentos que resumem a classificação. Nessa perspectiva, a OMS propôs uma lista genérica criada a partir da CIF, contendo as condições mais importantes a serem levantadas durante a atenção ao paciente. Essa checklist é composta por 152 categorias que representam os domínios mais relevantes da CIF e classifica 38 códigos de funções do corpo, 20 códigos de estrutura do corpo, 57 de atividade e participação e 37 códigos de fatores ambientais. ${ }^{9}$ Além desse instrumento, destaca-se o projeto de elaboração dos Core sets para algumas condições crônicas ${ }^{11}$. Esses core sets representam as principais categorias da classificação para determinadas doenças. Atualmente, um projeto multicêntrico internacional, coordenado pela Universidade de Munique, em fase de coleta de dados, tem o objetivo de validar os core sets elaborados para doze condições crônicas sendo o Brasil um dos centros participantes.

Um dos campos de estudo mais explorados para a aplicação da CIF tem sido a área de Medicina Física e Reabilitação, no que concerne ao acompanhamento do estado de 
saúde dos pacientes em tratamento ${ }^{12-15}$.

O uso da CIF tem sido considerado também na avaliação após transtornos agudos, condições traumáticas, condições crônicas e na geriatria ${ }^{16,17}$.

Alguns autores argumentam que a CIF deve ser explorada como uma base de informações padronizadas em estudos sobre incapacidade profissional, quando há necessidade de relacionar dados de saúde ocupacional com os de previdência social ${ }^{18,19}$.

Na Saúde Pública, tradicionalmente pouca atenção tem sido dada às pessoas com deficiência. No entanto, ao lado de recentes engajamentos por parte de organizações e de alguns governos, a publicação do modelo da CIF fornece as bases para as políticas e disciplinas da Saúde Pública em relação à população que apresenta deficiências ${ }^{20}$. Assim, uma das possibilidades de usos da CIF pode ser a contribuição para responder a importantes questões de Saúde Pública,tais como: qual é o estado de saúde das pessoas com deficiência comparadas às demais; que necessidades e que tipos de intervenções são mais adequadas para reduzir condições secundárias e promover a saúde das pessoas com deficiências, entre outras.

\section{Alguns dilemas colocados para a incorporação do modelo da CIF}

Até o presente, apesar do interesse pela adoção do modelo da CIF, existem poucos estudos em curso sobre a avaliação do seu impacto na atenção à saúde. Isso decorre de ser uma classificação recente, complexa e que apresenta certo grau de dificuldade em sua utilização. Do ponto de vista prático, sua aplicação requer um tempo muitas vezes maior do que a própria consulta, além dos aspectos inerentes à mudanças de conduta por parte dos profissionais da área da saúde.

Mesmo contribuindo para o processo de integração entre diversos setores da saúde, social e da pedagogia, sua compreensão teórica, incorporação e aplicabilidade prática têm refletido essas dificuldades. Um dos dilemas apresentados, segundo IMRIE (2004), diz respeito ao esclarecimento do seu papel biopsicossocial e do significado e implicações da universalização como um princípio para orientar o desenvolvimento de políticas em relação às pessoas com deficiências.

Outra questão apontada concerne à classificação de atividades e participação. Se diferentes estratégias de códigos são utilizadas, uma vez que existe uma variabilidade de apreciação dependente do contexto sociocultural, isso coloca dificuldades para a comparação de dados entre os países; assim, alguns países têm desenvolvido distinções próprias de atividade e de participação $0^{22,23}$.

$\mathrm{O}$ reconhecimento do papel central do meio ambiente no estado funcional dos indivíduos, agindo como barreiras ou facilitadores no desempenho de suas atividades e na participação social, mudou o foco do problema da natureza biológica individual da redução ou perda de uma função e/ ou estrutura do corpo para a interação entre a disfunção apresentada e o contexto ambiental onde as pessoas estão inseridas. Dessa forma, o modelo da CIF deverá ser investigado nas suas dimensões sociais, políticas e culturais, o que constitui um desafio para os Sistemas. No entanto, será mais adequado à medida que for utilizado por um número maior de profissionais, em locais diversos e a partir de pessoas e realidades diferentes.

A incorporação do uso da CIF nas práticas de atenção à saúde, tendo em vista que se trata da incorporação de uma nova tecnologia, embora já venha sendo adotada por diversos setores e equipes multidisciplinares, deve ser ainda amplamente explorada em relação à sua aceitabilidade $\mathrm{e}$ validade em diferentes áreas; seu impacto nos cuidados de saúde; seu potencial em medir o estado funcional dos pacientes e seu uso pelos sistemas de informação para elaboração de estatísticas de saúde ${ }^{24}$. Outro campo diz respeito às legislações pertinentes e à implementação de políticas públicas para as pessoas com deficiência.

Recomenda-se que estudos adicionais sejam realizados por profissionais e pesquisadores de diversas disciplinas e setores da saúde, incluindo também a participação das organizações da sociedade civil. 


\section{Referências}

1. [WHO] World Health Organization. The WHO Family of International Classifications. Disponível em: URL < http://www.who.int/classifications/en> [2005 May 27].

2. Organização Mundial de Saúde. $C I D-10$, tradução do Centro Colaborador da OMS para a Classificação de Doenças em Português. 9 ed. Rev -São Paulo: EDUSP, 2003.

3. Secretariado Nacional de Reabilitação, Ministério do Emprego e da Segurança Social. Classificação Internacional das Deficiências, Incapacidades e Desvantagens (handicaps). Lisboa; 1989.

4. Buñuales MTJ, Diego PG, Moreno JMM. La Clasificación Internacional del Funcionamento de la Discapacidad y de la Salud (CIF) 2001. Rev Esp Salud Publica 2002; 76: 271-9.

5. [WHO] World Health Organization. International Classification of functioning, disability and health: ICF. World Health Organization; 2001.

6. [OMS] Organização Mundoal da Saúde, CIF: Classificação Internacional de Funcionalidade, Incapacidade e Saúde [Centro Colaborador da Organização Mundial da Saúde para a Família de Classificações Internacionais, org.; coordenação da tradução Cassia Maria Buchalla]. São Paulo: Editora da Universidade de São Paulo - EDUSP; 2003.

7. Battistella LR, Brito CMM. Tendência e Reflexões: Classificação Internacional de Funcionalidade (CIF). Acta Fisiátrica 2002; 9(2): 98-101

8. Halbertsma, J. The ICIDH: health problems in a medical and social perspective. Disability and Rehabilitation 1995; 17(3/4): 128-34 (8).

9. [WHO]World Health Organization.Towards a Common Language for Functioning, Disability and Health - ICF. [WHO/EIP/GPE/CAS/01.3] Genebra; 2002.

10. Gray DB, Hendershot GE. The ICIDH-2: Developments for a new era of outcomes research. Arch Phys Rehabil 2000; 81(2): S10 - S14 (9).

11. Weigl M, Cieza A, Andersen C, Kollerits B, Amann E, Stucki G Identification of relevant ICF categories in patients with chronic health conditions: a delphi exercise. J Rehabil Med 2004; Suppl 44: 12-21.

12. Stamm TA, Cieza A, Machold KP, Smolen JS, Stucki G. Content comparison of occupation-based instruments in adult rheumatology and musculoskeletal rehabilitation based on the International Classification of Functioning, Disability and Health. Arthritis Rheum 2004; 51(6): 917- 24.

13. Mayo NE, Poissant L, Ahmed S, Finch L, Higgins J, Salbach NM et al. Incorporating the International Classification of Functioning, Disability and Health (ICF) into an electronic health record to crate indicators of function: proof of concept using the SF-12. J Am Med Inform Assoc 2004; 11(6): 514-22.
14. Arthanat S, Nochajski SM, Stone J. The international classification of functioning, disability and health and its application to cognitive disorders. Disabil Rehabil 2004 ; 26(4): 235-45.

15. Rentsch HP, Bucher P, ommen NI, Wolf C, Hefti H, Fluri E et al. The implementation of the International Classification of Functioning, Disability and Health (ICF) in daily practice of neurorehabilitation: an interdisciplinary project at the Kantonsspital of Lucerne, Switzerland. Disabil Rehabil 2003 ; 25(8): 411-21.

16. Stucki G, Ewert T, Cieza A. Value and application of the ICF in rehabilitation medicine. Disabil Rehabil 2002: 24(17): 932-8.

17. Cieza A, Brockow T, Ewert T, Amman E, Kollerits B, Chatterji S. Üstun TB, Stucki G. Linking Health-status measurements to the international classification of international classification of functioning, disability and health. J Rehabil Med 2002; 34: 205-10.

18. Willems H, De Kleijn-De Vrankrijker M. Work disability in the Netherlands: data, conceptual aspects, and perspectives. J Occup Environ Med 2002; 44(6): 510-5.

19. Sjögren-Rönka T, Ojanen MT, Leskinen EK, Tmustalampi S, Mälkiä, EA. Physical and Psychosocial prerequisites of functioning in relation to work ability and general subjective well-being among office workers Jogren-Ronka T, Ojanen MT, Leskinen E*, Leskinen EK. Jõgren-Rönka T, Ojanem MT, Leskinen EK, Tmustalampis.

20. MÄLKIÄ EA. Physical and psychosocial prerequisites of functioning in relation to work ability and general subjective well-stair among office par to work ability and general subjective well-being among office workers. Scand J Work Environ Health 2002; 28(3): 18490.

20. LOLLAR, DJ. Public Health and Disability: Emerging Opportunities. Public Health Reports, March-April 2002; 117: 131-6.

21. Imrie R. Demystifying disability: a review of the International Classification of Functioning, Disability and Health. Social Health IIIn 2004; 26(3): 287 -305.

22. Peremboom RJ, Chorus Erembooom RJ, Chorus A.Erenboom RJ, Chorus Ameremboom RJ, Chorus, AMR: mediar a ersidERENBOOM RJ, CHORUS AM. Measuring participation according to the International Classification of Functioning, Disability and Health (ICF). Disabil Rehabil 2003; ST 2003; 25(11-12): 577-87.

23. Dahl TH. International classification of functioning, disability and health: an introduction and discussion of its potential impact on rehabilitation services and research. $v$ 2002; 34 (5): 201-4.

24. Buchalla CM. A Classificação Internacional de Funcionalidade, Incapacidade e Saúde. Acta Fisiátrica, 2003; 10 (1): 29-31.

recebido em: 04/04/05

versão final reapresentada em: 31/05/05 aprovado em: 13/06/05 